\title{
Desigualdades de gênero e raça na pandemia de Covid-19: implicações para o controle no Brasil
}

\author{
Gender and race inequalities in the Covid-19 pandemic: implications \\ for control in Brazil
}

Ana Paula dos Reis $\mathbf{1 , 2}$, Emanuelle Freitas Góes $\mathbf{2 , 3}$, Flávia Bulegon Pilecco ${ }^{\mathbf{2} 4}$, Maria da Conceição Chagas de Almeida $\mathbf{2 , 5}^{\mathbf{5}}$ Luisa Maria Diele-Viegass,6, Greice Maria de Souza Menezes ${ }^{\mathbf{1}, \mathbf{2}}$, Estela M. L. Aquino 1,2

DOI: 10.1590/0103-11042020E423

1 Universidade Federal da Bahia (UFBA), Instituto de Saúde Coletiva (ISC) Salvador (BA), Brasil.

paula@ufba.br

2 Rede CoVida - Ciência, Informação e Solidariedade - Salvador (BA), Brasil.

${ }^{3}$ Fundação Oswaldo Cruz (Fiocruz), Centro de Integração de Dados e Conhecimentos para a Saúde (Cidacs) - Salvador (BA), Brasil.

4 Universidade Federal de Minas Gerais (UFMG),

Faculdade de Medicina, Departamento de Medicina Preventiva e Social - Belo Horizonte (MG), Brasil.

5 Fundação Oswaldo Cruz (Fiocruz), Instituto Gonçalo Moniz - Salvador (BA), Brasil.

6 Universidade de Maryland, Departamento de Biologia - College Park, Maryland, Estados Unidos da América.
RESUMO Esta revisão narrativa sintetizou evidências científicas sobre desigualdades de gênero e raça na pandemia de Covid-19, enfocando o trabalho produtivo/reprodutivo das mulheres, a violência de gênero e o acesso aos Serviços de Saúde Sexual e Reprodutiva (SSR). Os resultados confirmam que as desigualdades sociais devem ser consideradas para o efetivo controle da pandemia e para a preservação de direitos. Para além dos efeitos diretos do SARS-CoV-2, discute-se que barreiras de acesso a serviços de SSR podem ocasionar o aumento de gravidezes não pretendidas, abortos inseguros e mortalidade materna. O distanciamento social tem obrigado muitas mulheres a permanecer confinadas com seus agressores e dificultado o acesso a serviços de denúncia, incorrendo no aumento da violência de gênero e em desfechos graves à saúde. Como principais responsáveis pelo cuidado, as mulheres estão mais expostas a adoecer nas esferas profissional e doméstica. A conciliação trabalho-família tornou-se mais difícil para elas durante a pandemia. A literatura naturaliza as diferenças de gênero, raça e classe, com ênfase em fatores de risco. Uma agenda de pesquisa com abordagem interseccional é necessária para embasar a formulação de políticas que incorporem os direitos humanos e atendam às necessidades dos grupos mais vulneráveis à Covid-19.

PALAVRAS-CHAVE Gênero e saúde. Raça e saúde. Covid-19.

ABSTRACT This narrative review synthesized scientific evidence on gender and race inequalities in the Covid-19 pandemic, focusing on women's productive/reproductive work, gender-based violence, and the access to Sexual and Reproductive Health Services (SRHS). The results demonstrated that social inequalities must be considered for the effective control of the pandemic and for the preservation of rights. Besides the direct effects of SARS-CoV-2, the literature discusses that barriers to access SRHS can lead to an increase in unintended pregnancies, unsafe abortions, and maternal mortality. Also, social distancing has led several women to stay confined with their aggressors, which hinders the access to reporting services, incurring in the increase of gender-based violence and severe outcomes to health. As main responsible for the care, women are more prone to getting the virus in both professional and domestic spheres. The conciliation between work and family has become more difficult for them during the pandemic. Literature naturalizes gender, race, and social class differences, emphasizing risk factors. An intersectional research plan is needed to support the making of public policies that incorporate human rights and meet the needs of the most vulnerable to Covid-19.

KEYWORDS Gender and health. Race and health. Covid-19. 


\section{Introdução}

Desde sua emergência, em dezembro de 2019, a pandemia de Covid-19 tem se espalhado rapidamente, constituindo-se na maior crise sanitária dos últimos cem anos. Na ausência de vacina e de terapêuticas específicas, os esforços para seu controle têm implicado a adoção de medidas tradicionais de saúde pública, como o isolamento de casos, a quarentena de contatos e o distanciamento social, de modo a diminuir a transmissão do SARS-CoV-2 ${ }^{1}$. Essas medidas têm tido um profundo impacto na vida das pessoas, mas não atingem a todas da mesma forma e, ao que parece, vêm resultando no aprofundamento de desigualdades sociais prévias².

Em situações recentes de Emergência em Saúde Pública, como as epidemias de ebola e zika, constatou-se a prioridade em respostas biomédicas com a negligência das inequidades estruturais como as de gênero e raça ${ }^{\mathbf{3 , 4}}$, o que foi denominado de "tirania do urgente"5(357). Entre outros problemas invisibilizados, registraram-se o aumento da violência baseada em gênero e a desativação dos serviços de atenção à Saúde Sexual e Reprodutiva (SSR), com o aumento de gravidezes não pretendidas, aborto inseguro, mortalidade materna e Infecções Sexualmente Transmissíveis (IST). A experiência de epidemias anteriores motivou recomendações de organismos multilaterais ${ }^{6}$, com um alerta precoce do Fundo das Nações Unidas para a População (UNFPA) ${ }^{7}$ e da Organização Mundial da Saúde (OMS) ${ }^{8}$.

Outro aspecto resultante de normas de gênero, habitualmente naturalizado, é o papel privilegiado (quando não exclusivo) das mulheres como cuidadoras ${ }^{9}$, nos âmbitos profissional e doméstico, o que as torna mais vulneráveis ao contato com pessoas doentes e mais expostas às infecções.

Na pandemia de Covid-19, em países como o Brasil, marcados por imensas desigualdades sociais, configura-se um difícil cenário para o cumprimento das medidas para redução da mobilidade social e controle da doença ${ }^{\mathbf{1}}$. Gênero, raça e classe social são eixos estruturantes que organizam a vida social e condicionam os padrões de adoecimento, morte e acesso aos serviços, especialmente em situações de crise sanitária²

Este artigo buscou sistematizar evidências científicas sobre desigualdades de gênero e raça/etnia em tempos de Covid-19 e enfocar o trabalho produtivo/reprodutivo das mulheres, a violência de gênero no distanciamento social e o acesso aos serviços de SSR. Entende-se 'desigualdades' como a dimensão empírica das diferenças em coletividades humanas, reservando 'inequidades' como expressão da ausência de justiça que produz desigualdades evitáveis e desnecessárias ${ }^{10}$. Pretendeu-se, assim, subsidiar, no Brasil, gestores, profissionais de saúde e sociedade para o adequado controle da pandemia e para a proteção de grupos em maior vulnerabilidade social.

\section{Material e métodos}

Esta revisão narrativa partiu da triagem de artigos publicados de $1^{\circ}$ de dezembro de 2019, ao início da pandemia até 24 de julho de 2020, no LitCovid"1 um hub que concentra referências internacionais sobre Covid19, publicadas no PubMed. Definiram-se as seguintes estratégias de buscas para os temas de interesse: desigualdades de gênero, raça/ etnia e interseccionalidade (gender $O R$ race $O R$ racial OR racism OR ethnicity OR interseccionality); atenção à SSR (("health services") AND ("sexual" OR "reproductive" OR "contraceptives” OR "family planning” OR HIV OR “abortion")); violência de gênero ("violence against women" OR "gender violence" $O R$ "gender based violence" OR "domestic violence" OR "intimate partner violence" OR "domestic conflicts"); trabalho produtivo ("health professionals" OR "health care workers" OR "health workers" OR occupational health" OR occupation OR workers) e trabalho reprodutivo ("unpaid care work" OR "unpaid work" OR housework OR childcare OR "work-family" OR "caring for family"). Para cada conjunto, as autoras 
procederam à revisão de títulos e resumos, independentemente do tipo de publicação, conferindo-se prioridade aos artigos originais e de revisão, mas incluindo outros tipos (editorial, comentário, correspondência) cujo conteúdo avaliou-se como relevante. Foram incluídos os idiomas: português, inglês, espanhol e francês. Eventualmente, foram incorporadas referências cruzadas, além de documentos oficiais de organismos multilaterais engajados no enfrentamento da pandemia de Covid-19.

Ao final, foram selecionados 82 artigos, cujas leitura e análise foram sistematizadas segundo os temas definidos para a busca e apresentados a seguir.

\section{Resultados}

A produção científica sobre Covid-19 tem apresentado crescimento exponencial, o que se expressa no grande número de referências disponíveis ao início da presente revisão. Em 24 de julho de 2020, na base consultada, foram identificados 32.369 títulos, quase metade dos quais (43\%) abordava aspectos ligados ao diagnóstico clínico, laboratorial ou radiológico, a relatos de casos clínicos, procedimentos terapêuticos e desenvolvimento de vacinas. Apenas $2 \%$ das referências contemplavam gênero, raça/etnia ou interseccionalidade (dados não apresentados).

O corpus analisado reuniu 3.318 referências, entre as quais $56 \%$ tratavam da atenção à SSR, cuja maioria enfocava a atenção à gravidez e ao nascimento. Temáticas como HIV e IST aparecem na busca, mas isso decorre, em grande parte, pelo interesse em drogas usadas para o manejo dessas infecções virais, uma vez que podem ter efeitos sobre o SARS-CoV-2. Contracepção e aborto são temas pouco visíveis quando comparados à atenção pré-natal, ao parto e ao pós-parto. A identificação dessa lacuna motivou a ênfase no presente artigo na revisão sobre o planejamento reprodutivo e aborto, com breve menção aos serviços de pré-natal, parto e puerpério. Os artigos sobre violência doméstica contra as mulheres constituíram uma parcela diminuta (apenas 48 artigos), mas muito específica como resultado da sintaxe adotada. Na pandemia, o tema é abordado como relacionado com as estratégias de distanciamento social e, em alguns casos, associado a problemas de natureza psíquica em função do confinamento da população em suas residências, sem precedente na história.

Cerca de $43 \%$ do corpus examinado diz respeito ao trabalho profissional, com grande ênfase nos profissionais de saúde, pelo seu papel estratégico no tratamento das complicações da doença e na oferta dos serviços de saúde. O trabalho reprodutivo e o cuidado familiar não remunerado de crianças, idosos e doentes, exercido majoritariamente pelas mulheres, suscitam pouco interesse, sendo localizadas apenas 21 referências. A seguir, são apresentados os resultados desta revisão, que não pretendeu fornecer uma descrição detalhada e exaustiva da literatura, mas apreciá-la criticamente a partir da experiência e reflexão das autoras ${ }^{\mathbf{1 2}}$, para identificar lacunas e questões a serem aprofundadas em estudos futuros.

\section{Potenciais impactos da pandemia na atenção à saúde sexual e reprodutiva}

A Covid-19 traz consigo impactos à saúde que não se restringem àqueles causados diretamente pelo SARS-CoV-2, mas que decorrem de restrições ao acesso aos demais serviços de saúde pela priorização do controle da pandemia. Desde seu surgimento, pesquisadoras e organismos internacionais têm alertado para a possibilidade de descontinuidade da atenção em SSR.

Bloqueios nas cadeias de distribuição de contraceptivos, decorrentes do fechamento de fronteiras e outras restrições às movimentações para o controle da Covid-19, adotadas em países asiáticos, que são seus principais produtores, podem resultar na escassez desses insumos no mercado. Há possibilidade de interrupção da fabricação de contraceptivos farmacológicos e seus componentes ${ }^{13}$. A oferta desses serviços pode ser afetada pela 
suspensão de atendimentos eletivos de saúde e desvio de recursos humanos e financeiros para a contenção da pandemia' ${ }^{\mathbf{1 4}}$. Além disso, tal como ocorreu na epidemia da H1N1, pode haver redução do número de profissionais de saúde em função de seu adoecimento ${ }^{15}$. Essa restrição da oferta de serviços de SSR tende a ser mais grave em áreas de maior vulnerabilidade $^{13}$. Somam-se a isso fatores que podem reduzir a procura pelos serviços de SSR, como a diminuição da renda ocasionada pela recessão econômica e o receio de usuárias de busca-rem esses serviços por medo de contaminação em transportes públicos ou nas unidades de saúde ${ }^{16,17}$.

Dados da International Planned Parenthood Federation (IPPF) ${ }^{18}$ evidenciam que, até 9 de abril de 2020, 5.633 de seus centros de atendimento de planejamento reprodutivo haviam sido fechados em 64 países por causa da pandemia de Covid-19, o que representa $14 \%$ do total de pontos de entrega de anticoncepcionais da IPPF em 2018. Entre os serviços que permaneceram abertos, houve redução de testes de HIV, de assistência contraceptiva, de combate à violência de gênero e de aborto legal. Outros enfrentavam dificuldades em receber suprimentos, devido a atrasos na movimentação de mercadorias dentro dos países e à escassez de contraceptivos e de medicamentos para o HIV $^{18}$. Esse padrão de declínio do acesso aos serviços pode ter graves consequências, como o aumento de gravidezes não pretendidas, dos abortos inseguros e da mortalidade materna ${ }^{19}$.

A partir das Pesquisas Demográficas de Saúde, Riley e colaboradores ${ }^{13}$ estimaram o impacto da interrupção dos serviços essenciais de SSR na pandemia em países de baixa e média renda. Previram um declínio de $10 \%$ no uso de contraceptivos reversíveis de ação curta e longa, com mais de 48,5 milhões de mulheres com demandas por contracepção não atendidas e 15,4 milhões de gravidezes indesejadas adicionais.

Embora seja escassa a literatura, no Brasil e em outros países de baixa e média renda, registrou-se um maior risco de mortes de mulheres com Covid-19 durante o ciclo gravídico puerpe$\mathrm{ral}^{20}$, de forma distinta ao que ocorreu na Ásia e na Europa. Apenas um estudo na Suécia ${ }^{21}$ havia constatado maior probabilidade de gestantes com Covid-19 necessitarem de cuidados intensivos. Nos Estados Unidos da América (EUA $)^{22}$, casos de near miss relacionados com a doença foram registrados. No Brasil, uma análise de casos de Síndrome Respiratória Aguda Grave identificou 124 mortes durante a gravidez e no pós-parto entre fevereiro e junho ${ }^{23}$. Grávidas com comorbidades são mais afetadas, e há indícios de que essas mulheres enfrentaram barreiras de acesso à terapia intensiva ${ }^{24}$. Embora não haja dados nacionais, a literatura internacional tem mostrado que mulheres negras são mais infectadas ${ }^{25}$ e necessitam mais de internação por Covid-1926.

Para reduzir os óbitos maternos, o UNFPA recomenda que parturientes com Covid-19 devem ser atendidas em locais que assegurem cuidados respiratórios, e que grávidas com outras doenças respiratórias devem ser tratadas como prioridade e separadas das gestantes infectadas ${ }^{27}$. O aumento da mortalidade materna nesta pandemia requer um esforço especial da vigilância epidemiológica municipal e estadual, com reforço do papel dos Comitês de Morte Materna, para investigação dos óbitos de mulheres em idade fértil, das mortes maternas e de casos de near miss, com elucidação da sua relação com a Covid-19 e dos subgrupos de gestantes mais atingidas.

Publicações de entidades de ginecologia e obstetrícia têm recomendado a garantia dos serviços de pré-natal, de forma a proteger as gestantes da infecção pelo SARS-CoV-2. Devem ser adotados esquemas alternativos de acompanhamento, como consultas domiciliares, telessaúde ou por WhatsApp, para identificar as sintomáticas e assegurar a atenção às infectadas, especialmente gestantes de alto risco e profissionais de saúde, mais expostas à epidemia ${ }^{27}$. Protocolos de atenção ao parto e pós-parto orientam o manejo clínico das pacientes, o respeito à escolha do tipo de parto - na ausência de indicação técnica de 
cesárea - e a presença de acompanhante de preferência da mulher, guardada a proteção de todos os envolvidos ${ }^{\mathbf{2 8}}$.

Há evidências de que a infecção por SARSCoV-2 pode influir nos desfechos gestacionais, ao contrário do que indicavam os estudos iniciais de que o risco de complicações da doença entre grávidas não superava o encontrado na população adulta em geral29. Novas investigações, com metodologias mais sofisticadas e com grupos de comparação, começam a evidenciar um risco aumentado de complicações graves entre gestantes, possivelmente associadas a alterações fisiológicas da gravidez, além da transmissão vertical e de danos ao feto, sobretudo nos primeiros meses da gestação ${ }^{\mathbf{2 1}}$.

Quanto à atenção ao aborto, estudos indicam um aumento na procura pelos serviços após o início da pandemia ${ }^{30}$, o que pode decorrer da falta de acesso à contracepção e das incertezas quanto às consequências do SARS-CoV-2 sobre os desfechos reprodutivos ${ }^{30}$. Em meio à crise sanitária, a legitimidade desses serviços tem sido questionada em países com legislações restritivas e posições mais conservadoras em relação ao procedimento ${ }^{31,32}$. A pretexto de priorizar o controle da pandemia, recursos financeiros e humanos têm sido desviados e o aborto, adiado sob a alegação de que se trata de procedimento eletivo ${ }^{33}$, desconhecendo-se que sua concretização é tempo dependente.

Na maioria dos países latino-americanos, a legislação é conservadora, e o sistema de saúde não prioriza as mulheres, o que se agrava com a pandemia. No Chile e no Brasil, as iniciativas estão focadas na atenção à violência doméstica durante o distanciamento social, sem abordagem aos direitos reprodutivos. Na Argentina, ao contrário, governos de províncias, tanto de esquerda como de direita, mobilizaram-se para garantir os direitos sexuais e reprodutivos das mulheres, assegurando o acesso aos anticoncepcionais e ao aborto legal ${ }^{34}$.

No Brasil, com a grave crise política e a posição negacionista do governo federal, as iniciativas de proteção dos direitos das mulheres têm partido de organizações da sociedade civil, como a Rede Médica pelo Direito de Decidir ${ }^{35}$. O Ministério da Saúde (MS) demorou a assumir uma posição oficial sobre o tema, e o atendimento às mulheres durante a pandemia reduziu-se ao ciclo gravídico puerperal. Em $1^{\circ}$ de junho, foi lançada uma Nota Técnica, que reconhecia como essenciais os SSR, entre eles os de aborto seguro para os casos previstos em lei, e reiterava a importância da continuidade desses serviços ${ }^{36}$. Três dias depois, o MS desautorizou o documento e exonerou os coordenadores de Saúde da Mulher e de Saúde do Homem. Houve, em resposta, a manifestação de diversas organizações da sociedade civil - entre outras, a Federação Brasileira de Ginecologia e Obstetrícia, o Centro Brasileiro de Estudos de Saúde (Cebes) e a Associação Brasileira de Saúde Coletiva (Abrasco).

Apesar da centralidade do MS na crise política, seu corpo técnico e a pressão do controle social do sistema de saúde têm assegurado, não sem conflitos, a implementação de iniciativas relacionadas com a atenção à SSR. O uso da telessaúde foi autorizado e regulamentado, em caráter de excepcionalidade, durante a pandemia, pelo MS (Portaria $n^{\circ} 467$, de 20 de março de 2020) e pelo Conselho Federal de Medicina (Ofício n ${ }^{\circ} 1.756,19$ de março 2020), o que pode beneficiar consultas para mulheres com acesso a essas tecnologias.

Não se dispõe de informações sobre a oferta de contraceptivos na rede privada ou pública do País. Entretanto, estimam-se maiores dificuldades entre usuárias dos serviços públicos, já que, antes da epidemia, essas mulheres enfrentavam problemas pela disponibilidade limitada de métodos de longa duração, a exemplo do DIU (Dispositivo Intrauterino) com cobre e ausência daqueles com hormônio ${ }^{37}$. A redução dos serviços, a limitação de funcionamento de algumas unidades com adiamento e cancelamento de consultas podem dificultar o acesso, particularmente, daquelas mais jovens e de grupos de maior exclusão social que dependem do Sistema Único de Saúde (SUS).

Em relação ao aborto, há indicações de que as mulheres têm encontrado dificuldades em ter acesso a esses serviços no Chile ${ }^{38}$, na 
Bolívia $^{34}$ e no Equador ${ }^{34}$. No Brasil, houve a suspensão temporária da oferta de interrupções legais da gestação pelo Hospital Pérola Byington, de 26 a 30 de março, retomada após intervenção do Ministério Público e da Defensoria Pública do Estado de São Paulo. Um levantamento de organizações feministas indicou que apenas $55 \%$ dos 76 hospitais que realizavam abortos legais em 2019 estava em funcionamento durante a pandemia ${ }^{39}$.

\section{Uma emergência na emergência: violência doméstica contra as mulheres e Covid-19}

Tão logo se alastrou pelo mundo, a pandemia de Covid-19 provocou o acirramento de um problema crônico: a violência doméstica contra as mulheres perpetrada pelo parceiro íntimo ${ }^{40,41}$. De acordo com a OMS, esse tipo de violência triplicou durante a pandemia ${ }^{\mathbf{4 2}}$.

Em diversos países, as mulheres estão mais expostas à violência física ou sexual. Aproximadamente um terço de todas as mulheres que estiveram em um relacionamento sofreram violência por seu parceiro íntimo, sendo o ambiente doméstico o espaço de maior ocorrência; mais de um terço dos homicídios de mulheres são cometidos por um parceiro íntimo ${ }^{42}$. No Brasil, dos 3.739 homicídios de mulheres em 2019, 35\% foram tipificados como feminicídios ${ }^{\mathbf{4 8}}$, e o perfil do agressor segue o padrão global: majoritariamente, companheiros e ex-companheiros.

Durante emergências humanitárias e sanitárias, observa-se aumento da violência contra as mulheres, um reflexo das inequidades de gênero ${ }^{43}$. Em períodos pós-catástrofes naturais, como o furacão Katrina, no EUA, e o tsunami no Sri Lanka ${ }^{40,44-47}$, a violência doméstica contra as mulheres aumentou de modo expressivo. No Sudão do Sul, após décadas de guerras, mais de $50 \%$ das mulheres sofreram violência por parceiro íntimo ${ }^{43}$. Epidemias passadas, a exemplo do ebola na África Ocidental, dos surtos de doenças do cólera no Haiti e do zika vírus apresentaram, igualmente, crescimento da violência doméstica $\mathbf{3 0}^{\mathbf{3 0 1}, \mathbf{4 5}}$, além da redução no financiamento para serviços de saúde pública especializados em violência baseada em gênero $\mathbf{4 1 , 4 5}^{\mathbf{4}}$.

A literatura nacional e internacional tem argumentado que o espaço doméstico nem sempre é um lugar seguro para as mulheres, principalmente para aquelas com histórico de violência doméstica e familiar ${ }^{\mathbf{4 6}, 48}$. A casa é, sabidamente, o espaço no qual ocorrem abusos físicos, psicológicos e sexuais contra as mulhe$\mathrm{res}^{\mathbf{4 5}}$, aspecto que se torna mais preocupante no contexto da pandemia, cuja estratégia mais eficaz para evitar a infecção pelo SARS-CoV-2, e sua disseminação, é manter-se em casa.

Nesse sentido, mulheres da maioria dos países têm lidado cotidianamente com um importante paradoxo: atender às recomendações das autoridades sanitárias de distanciamento social e, ao mesmo tempo, lidar com o maior risco de serem agredidas ou assassinadas pelos seus parceiros ${ }^{30}$.

As consequências das medidas de distanciamento social implementadas para contenção da pandemia são pouco conhecidas, sendo que os planos adotados pelos países para controle do SARS-CoV-2 não avaliaram o provável impacto psicológico nas populações ${ }^{44}$. Desde que a OMS decretou a epidemia de SARSCoV-2 como Emergência de Saúde Pública de Importância Internacional, observou-se um grau de confinamento das populações sem precedentes. A literatura sobre saúde mental aponta que medidas de cumprimento do distanciamento social são importantes preditores de estresse, com efeitos como distúrbios de sono, ansiedade, depressão e sintomas pós-traumáticos. A violência doméstica é abordada como uma consequência provável dessas medidas de controle da pandemia ${ }^{44}$.

A insegurança que caracteriza a vida das mulheres, em especial aquelas que vivem em uma relação abusiva com seus parceiros, acirrou desde o início da pandemia. A mídia nacional e internacional tem relatado que a violência doméstica, em todas as suas expressões, cresceu no período ${ }^{49}$. Nessa 
mesma direção, um indicador expressivo é o aumento de pesquisas relacionadas com a rede de apoio a mulheres em situação de violência doméstica no Google, que aumentou $75 \%{ }^{47}$. A literatura científica tem se debruçado sobre $o$ fenômeno e, na mesma direção das instituições multilaterais ${ }^{27,50}$ e da mídia, afirma que existe um expressivo aumento de denúncias de violência doméstica contra as mulheres perpetrada pelo parceiro íntimo em vários países do mundo 40,51-53.

A China apresentou os primeiros dados sobre o aumento da violência doméstica associada às medidas de contenção da Covid-19. Relatórios da província de Hubei indicaram que esses casos mais do que duplicaram durante o período de quarentena em relação ao mesmo período do ano anterior ${ }^{41,54}$.

No Reino Unido, a 'Linha Nacional de Atendimento ao Abuso Doméstico' registrou um aumento de $25 \%$ nas ligações e mensagens on-line após o início das medidas de distanciamento social51,55. A rede nacional italiana de abrigos para mulheres sujeitas à violência de gênero mostrou que 2.867 mulheres entraram em contato com 80 abrigos de 2 de março a 5 de abril de $2020^{54}$. Isso representa um aumento acentuado (74,5\%) quando comparado ao ano de 2018, com destaque para o fato de que um quarto das mulheres acessaram a rede pela primeira vez ${ }^{54}$.

Nos EUA, os dados disponíveis são limitados para avaliar como a violência doméstica mudou após a implementação das medidas de distanciamento social ${ }^{52}$. No entanto, o sistema de chamada 'Linha Direta Nacional de Violência Doméstica' informou que tem recebido muitas chamadas durante a pandemia ${ }^{46}$. Um estudo no campo do direito criminal evidenciou que os padrões de crime foram impactados pelas medidas de distanciamento social56. Os autores analisaram as chamadas diárias para os serviços policiais em Los Angeles e Indianópolis. Em ambos os locais, aumentaram as chamadas relativas à violência doméstica ${ }^{56}$. Outro estudo afirma que, também entre as mulheres veteranas, as queixas de violência doméstica cresceram ${ }^{57}$.
Na França, os registros de violência doméstica aumentaram $30 \%$ desde que o lockdown foi iniciado. Houve um aumento de $33 \%$ nas chamadas de linha de apoio em Cingapura. Após apenas uma semana de lockdown, a Comissão Nacional para as Mulheres na Índia registrou quase o dobro do número de reclamações. No entanto, as denúncias foram recebidas por e-mail. Considerando as mulheres que não têm acesso à internet, supõe-se que a ocorrência da violência doméstica deva ser significativamente maior ${ }^{55}$. Em Chipre, seguidas as solicitações para as pessoas ficarem em casa, observou-se aumento de mais de $30 \%$ de chamadas para os números da linha de apoio à violência ${ }^{51,52,55}$.

Na América Latina, Argentina, Colômbia, México e Brasil também registram aumento nas chamadas de emergência relacionadas com a violência doméstica. Na Argentina, as ligações aumentaram $25 \%$ desde o início do lockdown, em 20 de março ${ }^{52}$, e os crimes de feminicídio subiram $50 \%$, quando comparados ao mesmo período no ano anterior ${ }^{58}$. Na Colômbia, $91 \%$, e no México, $60 \%{ }^{59}$. O cenário brasileiro é semelhante e, de acordo com a Ouvidoria Nacional dos Direitos Humanos, do Ministério da Mulher, da Família e dos Direitos Humanos, o número de denúncias registradas pelos serviços Disque 100 e Ligue 180 subiu $18 \%$ entre os dias $1^{\circ}$ e 25 de março de $2020^{48}$.

Na Espanha, no entanto, observou-se um decréscimo de $40 \%$ nas denúncias. Ao contrário da suposição que se trata de uma redução dos casos de violência doméstica, argumenta-se que as medidas de isolamento e distanciamento social dificultaram a busca das vítimas por ajuda na rede de atenção ou a impossibilidade de comunicar-se por telefone com as autoridades policiais, na medida em que estavam em convivência integral com seus agressores 59,60 . Assim, estatísticas que apresentam diminuição das queixas de violência doméstica podem estar encobrindo efeitos e barreiras relacionados com estratégias de contenção da pandemia.

A exposição das mulheres também aumenta pela redução de acesso a serviços de apoio às 
vítimas, pela falta de conexão entre as redes institucionais (saúde, justiça, assistência), pela impossibilidade de deixar a residência e procurar um local mais seguro ou contar com ajuda

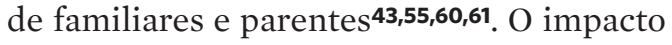
econômico da pandemia, incluindo o desemprego, o medo de a violência atingir os filhos e a dependência financeira do parceiro diminuem as chances de as mulheres romperem ou resistirem a relacionamentos abusivos ${ }^{43}$.

Assim como as instituições multilate-

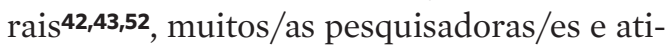
vistas de direitos humanos buscaram enfatizar a importância de mitigar os efeitos da violência doméstica no contexto da pandemia. As recomendações incluem o uso de estratégias on-line e telemedicina no atendimento médico, psicológico e social às mulheres $\mathbf{4 3 , 4 7 , 6 2}^{\text {, a manutenção }}$ de serviços de cuidados pós violência sexual ${ }^{41}$, a criação de novas formas de identificação de mulheres em situação de maior risco. Autores/as enfatizam o papel dos governos e profissionais de saúde, na adoção de formas alternativas para alcançar a população atingida ou em risco $0^{62,63}$. Ressalta-se, ainda, que a canalização de recursos para a prestação de serviços de emergência em resposta à Covid-19 tem colocado em risco a saúde de mulheres e meninas em todo o mundo ${ }^{43}$. Autoridades e organizações humanitárias devem reconhecer e tratar os efeitos das epidemias relacionados ao gênero ${ }^{\mathbf{4 1}}$.

\section{Cuidar em tempos de Covid-19 afeta a saúde das mulheres}

A crise sanitária desencadeada pela pandemia de Covid-19 teve um profundo impacto no mundo do trabalho. A alta transmissibilidade do SARS-CoV-2 e a identificação de clusters de transmissão nos locais de trabalho promoveram a adoção pelos países da suspensão de atividades não essenciais, revezamento ou flexibilização de jornadas e trabalho remoto em casa para funcionários públicos e trabalhadores do setor privado ${ }^{1}$. Em diversos países, foram adotados programas de proteção social no sentido de mitigar os efeitos econômicos da crise gerada pela pandemia. Em outros, como no Brasil, sob a justificativa de preservação de empregos e proteção das empresas ${ }^{64}$, tem havido a violação de direitos trabalhistas com a flexibilização da legislação vigente, de modo a permitir a suspensão do vínculo de trabalho, a redução da jornada de trabalho e de salários. As populações mais vulneráveis, em especial as mulheres, negras e pobres, estão mais sujeitas à demissão e ao corte de salários, aumentando o número de desempregados e a informalidade.

No Brasil, a chegada da pandemia de Covid19 se deu em um momento de aumento da pobreza e da extrema pobreza, que vinha ocorrendo desde 2015, revertendo a tendência decrescente de anos anteriores. Segundo o Instituto Brasileiro de Geografia e Estatística (IBGE), aumentou a proporção de trabalhadores informais e com vínculo precário (41,5\% da população ocupada), entre os quais, a maioria constituída de pretos e pardos ${ }^{65}$. Em que pese a tendência histórica de aumento da escolarização feminina e da entrada continuada das mulheres no mercado de trabalho, no momento prévio à chegada da Covid-19, persistia uma marcada divisão sexual do trabalho na sociedade brasileira. As mulheres contavam com um espectro mais reduzido de ocupações, ganhavam salários mais baixos e estavam mais sujeitas à informalidade ${ }^{65}$. Mantinham-se profundas desigualdades de gênero na esfera doméstica e, mesmo engajadas em jornadas extensas de trabalho profissional, permaneciam como as principais responsáveis pelo cuidado da casa e da família, dedicando, em média, 21,3 horas por semana a esse conjunto de atividades enquanto os homens, 10,9 horas ${ }^{65}$.

Em tempos de pandemia, a literatura internacional tem mostrado que essas desigualdades de gênero, que atribuem às mulheres o papel de cuidar, as colocam mais expostas a adoecer nas esferas profissional e doméstica, além de aprofundar inequidades sociais prévias ${ }^{66}$.

Elas são maioria entre os profissionais de saúde, em várias partes do mundo ${ }^{67}$ e no Brasil ${ }^{68}$, o que se acentua em ocupações como a enfermagem e naquelas de nível técnico e 
médio, em uma composição que é atravessada por gênero, raça e classe social. No exercício da profissão, estão mais expostas à infecção por SARS-CoV-2 e a literatura acumulada em diversos países, bastante expressiva, confirma o alto grau de contaminação dos/as profissionais de saúde ${ }^{69-73}$. Porém, essa literatura caracteriza-se pela naturalização das diferenças e não contempla a heterogeneidade desse grupo, marcado pela divisão técnica e pela divisão social do trabalho, às quais se sobrepõem relações de gênero, raça e classe social ${ }^{74,75}$.

Esse grupo profissional também apresenta mais problemas de saúde mental em decorrência do contato permanente com pessoas com complicações graves da Covid-19, em jornadas de trabalho extenuantes e preocupadas em não levar a infecção para suas famílias. Muitos artigos originais e de revisão, em diferentes países asiáticos e europeus, têm reunido evidências de que profissionais de saúde, especialmente as enfermeiras, enfrentam um alto grau de estresse, ansiedade, depressão, insônia devido à pandemia de Covid-1972,7679. Preocupações com o risco de contagiar a própria família aparecem como um importante fator de estresse, e isso é mais frequente entre as mulheres ${ }^{76}$. A maior prevalência de ansiedade e depressão, entre mulheres, e sua associação com o adoecimento por Covid-19 de amigos e parentes é descrita, não apenas entre profissionais de saúde, mas também na população geral em países tão distintos como a Turquia ${ }^{80}$ e a China ${ }^{81}$.

A maior exposição das mulheres a infecções como a Covid-19 não se explica apenas pela sua super-representação entre profissionais de saúde ${ }^{82}$. Isso se dá também pela segregação de gênero e raça ${ }^{83-85}$ : são as mulheres negras as mais inseridas em ocupações com menor prestígio e remuneração, no emprego doméstico e como cuidadoras de idosos e portadores de necessidades especiais, no trabalho informal e precário, dificultando o cumprimento da recomendação de distanciamento social e as colocando em situações de maior proximidade física com pessoas com Covid-19.
O teletrabalho ou trabalho remoto foi adotado em quase todos os países como forma de manter atividades nos setores público e privado e, ao mesmo tempo, permitir que os trabalhadores permanecessem em casa, reduzindo a mobilidade social e, consequentemente, a transmissão do SARS-CoV-2. Essa não é uma modalidade nova de trabalho, e há uma literatura razoável, prévia à pandemia, sobre seus efeitos de isolamento social e profissional e sua relação com desfechos negativos na saúde mental ${ }^{86}$. Ademais, ao borrar os limites entre vida profissional e privada, principalmente para as mulheres, o trabalho em casa pode representar um grande desafio durante a pandemia.

No desempenho de tarefas domésticas e familiares, historicamente a elas atribuídas, em tempos de distanciamento social, as mulheres têm se deparado com a diminuição do suporte institucional para o cuidado dos filhos, com o fechamento de escolas e creches e a educação das crianças em casa ${ }^{87}$. Isso se agravou com a impossibilidade de contar com as redes tradicionais de apoio de avós e mulheres mais velhas na família, que passou a ser não recomendado por estas integrarem o grupo de maior risco de complicações graves da Covid-1988.

O fechamento de escolas foi uma medida adotada por quase todos os países ${ }^{1,89}$ e se baseia em estudos com epidemias anteriores, que comprovaram seu impacto na redução da transmissão e do surgimento de novos casos. Um dos efeitos benéficos dessa medida é o decréscimo da mobilidade social dos pais, mas isso pode ter diferentes impactos para mulheres e homens em decorrência das desigualdades de gênero no cuidado da casa e de crianças. Particularmente para profissionais de saúde com filhos, as medidas podem ocasionar o absenteísmo em um setor essencial no curso da pandemia. Nesse sentido, a Áustria, a Holanda e a Inglaterra fecharam as escolas, exceto para filhos de trabalhadores de setores essenciais ${ }^{\mathbf{9 0}-92}$.

O trabalho doméstico foi redobrado pelas recomendações de higienização e limpeza de objetos, alimentos e superfícies, além da 
presença constante dos familiares em casa, para atender à recomendação de distanciamento social. Como tradicionais responsáveis pelos cuidados da saúde física e mental da família, tem sido a elas demandado o cuidado de parentes com necessidades especiais (idosos e portadores de deficiências) e de familiares com Covid-19 que não exigem internação, em muitas circunstâncias sem poder contar com o suporte de cuidadores profissionais, que, em sua maioria, também são mulheres ${ }^{\mathbf{8 8 , 9 3}}$.

Desse modo, seja no trabalho em atividades essenciais, seja no trabalho remoto, a conciliação de demandas profissionais e familiares, já tão complicada anteriormente, tornou-se uma difícil equação para as mulheres durante a pandemia. Embora seja escassa a literatura sobre os impactos dessa sobrecarga na saúde, em tempos de Covid-19, pode-se supor que sejam acentuados os efeitos descritos anteriormente de fadiga, ansiedade, depressão, distúrbios do sono, adoção de hábitos menos saudáveis (dieta menos saudável, diminuição da atividade física e descanso insuficiente).

\section{Considerações finais}

A presente revisão buscou sintetizar evidências científicas sobre as desigualdades sociais na pandemia de Covid-19, com ênfase nas de gênero e raça. Para isso, foi sistematizada uma produção científica, que tem crescido exponencialmente, mas tem grande ênfase nos aspectos clínicos e terapêuticos, com pequena expressão dos determinantes sociais e culturais envolvidos. Apresenta como limite o fato de que não foram incluídas outras fontes, mas tem como principal vantagem ser o PubMed a principal base de referências disponível, que ademais só inclui aquelas aprovadas por peer review ${ }^{\mathbf{1 1}}$. A revisão narrativa pode ser criticada pela falta de um protocolo rígido e pela escolha subjetiva e pessoal de referências. Entretanto, apresenta-se como alternativa adequada às estratégias estruturadas - como a revisão sistemática e a integrativa - quando se pretende explorar criticamente um tema ainda pouco conhecido $^{\mathbf{1 2}}$. Isso se baseia no entendimento de que a revisão narrativa permite identificar lacunas, desenvolver ideias e mapear aspectos, que poderiam ser obscurecidos pelas regras rígidas de inclusão e exclusão, e pela aplicação de critérios de qualidade. Ademais, tem a grande vantagem de ser realizada em tempo mais curto, o que se justifica pela urgência de respostas para o controle da pandemia.

Os resultados da presente revisão oferecem um panorama que contradiz o mito difundido sobre o caráter 'democrático' do SARS-CoV-2, apontando as desigualdades de gênero e raça que condicionam a ocorrência da Covid-19 e suas implicações para o cumprimento de medidas recomendadas para o seu controle.

Em epidemias anteriores, negligenciaram-se inequidades estruturais de raça e gênero e a fragilidade dos serviços de saúde. Esse padrão tem se repetido na pandemia de Covid-19, principalmente em países latino-americanos, que não têm reconhecido enquanto essenciais os serviços de SSR. O Brasil encontra-se em uma situação mais grave, considerando o desmonte de serviços de saúde e a perda de direitos conquistados, especialmente os sexuais e os reprodutivos. Pesquisas futuras devem avaliar a ocorrência de barreiras de acesso aos serviços e seus impactos sobre a SSR, considerando as desigualdades de gênero e raça em uma perspectiva interseccional.

Governos federal, estaduais e municipais devem promover o funcionamento dos SSR, considerados serviços essenciais na pandemia, com atendimento remoto quando possível, assegurando às mulheres a atenção ao planejamento familiar e ao pré-natal. Devem ser garantidos o acesso e a atenção oportuna às parturientes e seus recém-nascidos, bem como às mulheres com quadros de abortamento, com adoção de precauções para sua segurança. Deve-se também assegurar a atenção às gestantes sintomáticas para a Covid-19, com orientação às mulheres sobre serviços de referência.

A literatura consultada registra um aumento da violência doméstica, em vários países do 
mundo, e recomenda medidas de mitigação desse fenômeno. No entanto, as estratégias de contenção da pandemia, em geral, não contemplaram esse provável efeito. A realização de estudos futuros sobre o tema deve dimensionar o fenômeno e suas consequências na vida e no bem-estar das mulheres considerando os diversos contextos sociais.

Durante a pandemia, deve ser preservada a plena atuação das redes de atenção às mulheres em situação de violência, particularmente os serviços de aborto nos casos previstos em lei. É fundamental o atendimento imediato às mulheres com profilaxia às IST (Infecções Sexualmente Transmissíveis), contracepção de emergência, referência para apoio psicológico e legal, e acesso à interrupção segura da gravidez para as mulheres que assim o desejarem.

Em relação ao tema do trabalho profissional, embora exista uma robusta produção científica focada no trabalho em saúde, são mais escassos os conhecimentos sobre outras ocupações. Há uma ênfase nas normas de biossegurança, no uso de equipamentos de proteção individual e na higienização; todavia, é menos frequente a discussão sobre a organização e as condições gerais de trabalho e seus determinantes estruturais. A literatura naturaliza ou mesmo obscurece as diferenças de gênero, raça e classe, sendo predominante a epidemiologia de fatores de risco. As pesquisas sobre o trabalho reprodutivo e o cuidado familiar não pago são quase inexistentes, quando sua necessidade foi redobrada. Sua invisibilidade resulta na insuficiência de conhecimentos sobre os efeitos da sobrecarga laboral na saúde física e mental das mulheres, principais responsáveis pela sua realização.

Em que pese a identificação de artigos sobre desigualdades raciais e étnicas, assim como os estudos sobre gênero, essas duas vertentes ainda dialogam pouco. Abordagens interseccionais são incipientes, e muitas questões estão postas, exigindo a construção de uma forte agenda de pesquisa, com abordagem interseccional de gênero, raça e classe. Desse modo, a formulação de políticas será embasada por conhecimentos científicos que incorporem os direitos humanos - em especial, os sexuais e reprodutivos - e que atenda às necessidades dos grupos mais vulneráveis à Covid-19 e suas consequências.

\section{Colaboradoras}

Reis AP (0000-0002-6750-0187)*, Góes EF (0000-0001-9288-6723)* e Aquino EML (0000-0002-8204-1249)* contribuíram para a concepção do estudo e a estrutura inicial do manuscrito e para levantamento, seleção e análise das referências; e para elaboração, revisão crítica e aprovação do manuscrito (Góes EF compartilha com Reis AP igualmente a primeira autoria. Aquino EML ocupa a posição Sênior). Pilecco FB (0000-0001-8316-8797)*, Almeida MCC (0000-0002-4760-4157)*, Diele-Viegas LM (0000-0002-9225-4678)* e Menezes GMS (0000-0002-8393-2545)* contribuíram para levantamento, seleção e análise das referências, elaboração, revisão crítica e aprovação do manuscrito. 


\section{Referências}

1. Aquino EML, Silveira IH, Pescarini JM, et al. Social distancing measures to control the COVID-19 pandemic: potential impacts and challenges in Brazil. Cienc. Saúde Colet. 2020; 25(supl1):2423-46.

2. Ryan NE, El Ayadi AM. A call for a gender-responsive, intersectional approach to address COVID-19. Global Pub. Health. 2020; 15(9):1404-14.

3. Smith J. Gender matters in responding to major disease outbreaks like Ebola. Report from The Conversation [internet]. 2019 [acesso em 2020 mar 20]. Disponível em: https://reliefweb.int/report/world/ gender-matters-responding-major-disease-outbreaks-ebola.

4. Wenham C, Smith J, Morgan R. COVID-19: the gendered impacts of the outbreak. The Lancet. 2020; 395(10227):846-8.

5. Smith J. Overcoming the 'tyranny of the urgent': integrating gender into disease outbreak preparedness and response. Gender \& Development. 2019; 27(2):355-69.

6. Tolu LB, Feyissa GT. Guidelines and best practice recommendations on contraception and safe abortion care service provision amid COVID-19 pandemic: Scoping review [preprint] [internet]. 2020 [acesso em 2020 jun 9]. Disponível em: https://www.researchsquare.com/article/rs-25326/vl.

7. UNFPA Asia Pacific Regional Office. Coronavírus (2019-nCOV) guidance document 2020 [internet]. [acesso em 2020 jul 24]. Disponível em: https://china.unfpa.org/sites/default/files/pub-pdf/APRO\%20 Coronavirus\%202019-nCoV\%20Guidance\%20 Note-\%20final\%20\%2806\%20Feb\%202020\%29.pdf.

8. World Health Organization. COVID-19: Operational guidance for maintaining essential health services during an outbreak: WHO; 2020. [internet]. [acesso em 2020 jul 24]. Disponível em: https://www.who.int/publications-detail/covid-19-operational-guidance-for-maintaining-essential-health-services-during-an-outbreak.
9. Castellanos-Torres E, Mateos JT, Chilet-Rosell E. COVID-19 en clave de género. Gaceta Sanit. 2020; 34(5):419-21.

10. Almeida-Filho N. A problemática teórica da determinação social da saúde (nota breve sobre desigualdades em saúde como objeto de conhecimento). Saúde debate. 2009; 33(83):349-70.

11. Chen Q, Allot A, Lu Z. Keep up with the latest coronavirus research. Nature. 2020; 579(7798):193.

12. Jesson J, Matheson L, Lacey FM. Doing your literature review: Traditional and systematic techniques. London: Sage; 2013.

13. Riley T, Sully E, Ahmed Z, et al. Estimates of the Potential Impact of the COVID-19 Pandemic on Sexual and Reproductive HealthIn Low- and Middle-Income Countries. Inter. Perspec. Sex. Reprod. Health. 2020; 46:73-6.

14. Hussein J. COVID-19: What implications for sexual and reproductive health and rights globally? Sex. Reprod. Health Matters. 2020; 28(1):1746065.

15. Stephens I. Pandemic flu: implications for sexual and reproductive health services. J. fam. plann. Reprod. health care. 2009; 35(4):215-6.

16. Todd-Gher J, Shah PK. Abortion in the context of COVID-19: a human rights imperative. Sex. Reprod. Health Matters. 2020; 28(1):1-4.

17. Ahmed Z, Sonfield A. The COVID-19 Outbreak: Potential Fallout for Sexual and Reproductive Health and Rights 2020 [internet]. [acesso em 2020 jul 24]. Disponível em: https://www.guttmacher.org/article/2020/03/covid-19-outbreak-potential-fallout-sexual-and-reproductive-health-and-rights.

18. International Planned Parenthood Federation. COVID-19 pandemic cuts access to sexual and reproductive healthcare for women around the world: IPPF; 2020 [internet]. [acesso em 2020 jul 24]. Disponível 
em: https://www.ippf.org/news/covid-19-pandemic-cuts-access-sexual-and-reproductive-healthcare-women-around-world.

19. Bietsch K, Williamson J, Reeves M. Family Planning During and After the West African Ebola Crisis. Stud. Fam. Planning. 2020; 51(1):71-86.

20. Amorim MMR, Soligo Takemoto ML, et al. Maternal deaths with coronavirus disease 2019: a different outcome from low- to middle-resource countries? Am J Obstet Gynecol. 2020; 223(2):298-9.

21. Westgren M, Pettersson K, Hagberg H, et al. Severe maternal morbidity and mortality associated with COVID 19: The risk should not be down played. Acta Obstet Gynecol Scand. 2020; 99:815-6.

22. Breslin N, Baptiste C, Gyamfi-Bannerman C, et al. Coronavirus disease 2019 infection among asymptomatic and symptomatic pregnant women: two weeks of confirmed presentations to an affiliated pair of New York City hospitals. Am. J. Obstetrics Gynec. MFM. 2020; 2(2):100118.

23. Amorim MMR, Soligo Takemoto ML, Fonseca EBd. Maternal deaths with coronavirus disease 2019: a different outcome from low- to middle-resource countries? Am. J. Obstetrics Gynec. MFM. 2020: 223(2):298-299.

24. Takemoto MLS, Menezes MO, Andreucci CB, et al. The tragedy of COVID-19 in Brazil: 124 maternal deaths and counting. Int J Gynecol Obstet. 2020: ijgo.13300.

25. Knight M, Bunch K, Vousden N, et al. Characteristics and outcomes of pregnant women hospitalised with confirmed SARS-CoV-2 infection in the UK: a national cohort study using the UK Obstetric Surveillance System (UKOSS). [internet]. [acesso em 2020 jul 24]. Disponível em: http://medrxiv.org/lookup/doi/ 10.1101/2020.05.08.20089268.

26. Ellington S, Strid P, Tong VT, et al. Characteristics of Women of Reproductive Age with Laboratory-Confirmed SARS-CoV-2 Infection by Pregnancy Status -
United States, January 22-June 7, 2020. Morb. Mortal Wkly Rep. [internet]. 2020 [acesso em 2020 jul 14]; (69). Disponível em: https://www.cdc.gov/mmwr/ volumes/69/wr/mm6925al.htm.

27. Fundo de População das Nações Unidas Brasil. Saúde sexual e reprodutiva e direitos: contraceptivos modernos e outras necessidades de insumos médicos, inclusive para a prevenção, proteção e resposta a COVID-19: UNFPA Brasil [internet]. [acesso em 2020 jul 24]. Disponível em: https://brazil.unfpa.org/pt-br/publications/ sa\%C3\%BAde-sexual-e-reprodutiva-e-direitos-contraceptivos-modernos-e-outras-necessidades-de.

28. Trapani Júnior A, Vanhoni LR, Silveira SK, et al. Childbirth, Puerperium and Abortion Care Protocol during the COVID-19 Pandemic. Rev Bras Ginecol Obstet. $2020 ; 42(06): 349-55$.

29. Juan J, Gil MM, Rong Z, et al. Effect of coronavirus disease 2019 (COVID 19) on maternal, perinatal and neonatal outcome: systematic review. Ultrasound Obstet Gynecol. 2020; 56(1):15-27.

30. Tang K, Gaoshan J, Ahonsi B. Sexual and reproductive health (SRH): a key issue in the emergency response to the coronavirus disease (COVID-19) outbreak. Reprod. Health. 2020;17(1):59.

31. Tanne JH. Coronavirus pandemic stirs fight over abortion rights in US. BMJ. 2020; (369):m1733.

32. Berer M, Hoggart L. Progress toward Decriminalization of Abortion and Universal Access to Safe Abortions: National Trends and Strategies. Health Hum Rights. 2019; 21(2):79-83.

33. Bayefsky MJ, Bartz D, Watson KL. Abortion during the Covid-19 Pandemic - Ensuring Access to an Essential Health Service. N Engl J Med. 2020; 382(19):e47.

34. Bruno MM. Como vão os direitos reprodutivos na América Latina durante a pandemia do coronavírus. Gênero e Número [internet]. 2020 [acesso em 2020 jul 24]. Disponível em: http://www.generonumero.media/como-estao-os-direitos-reprodutivos-na-america-latina-em-tempos-de-coronavirus/. 
35. Rede Médica pelo Direito de Decidir. Nota Oficial: Pela manutenção dos programas de planejamento reprodutivo frente a pandemia da COVID-19 [internet]. [acesso em 2020 jul 24]. Disponível em: https:// assets-institucional-ipg.sfo2.cdn.digitaloceanspaces. com/2020/04/RedeMedicapeloDireitodeDecidir_ NotaPelaManutencaoProgramasPlanejamentoReprodutivo.pdf.

36. Brasil. Ministério da Saúde. Nota Técnica No 16/2020-COSMU/CGCIVI/DAPES/SAPS/MS. Brasília, DF: Ministério da Saúde; Secretaria de Atenção Primária à Saúde; 2020.

37. Ferreira-Filho ES, Melo NR, Sorpreso ICE, et al. Contraception and reproductive planning during the COVID-19 pandemic. Expert Review of Clinical Pharmacology. 2020; 13(6):615-22.

38. Boueri AG. Na pandemia, redes feministas se tornam ainda mais fundamentais para as mulheres que abortam na América Latina. Gênero e Número [internet]. [acesso em 2020 jul 24]. Disponível em: http://www. generonumero.media/pandemia-redes-feministas-aborto-america-latina/.

39. Silva VR, Ferreira L. Só $55 \%$ dos hospitais que ofereciam serviço de aborto legal no Brasil seguem atendendo na pandemia [internet]. Gênero e Número. 2020 [acesso em 2020 jun 2]. Disponível em: http:// www.generonumero.media/so-55-dos-hospitais-que-ofereciam-servico-de-aborto-legal-no-brasil-seguem-atendendo-na-pandemia/.

40. Kofman YB, Garfin DR. Home is not always a haven: The domestic violence crisis amid the COVID-19 pandemic. Psychol Trauma. 2020; 12(S1):S199-S201.

41. Roesch E, Amin A, Gupta J, et al. Violence against women during covid-19 pandemic restrictions. BMJ. 2020; (369):m1712.

42. UN Women. COVID-19 and Ending Violence Against Women and Girls. New York: United Nations Entity for Gender Equality and the Empowerment of Women (UN Women) [internet]. [acesso em 2020 jul 24]. Disponível em: https://www.unwomen.org/-/media/ headquarters/attachments/sections/library/publications/2020/issue-brief-covid-19-and-ending-violence-against-women-and-girls-en.pdf?la=en\&vs=5006.

43. John N, Casey SE, Carino G, et al. Lessons Never Learned: Crisis and gender-based violence. Dev World Bioeth. 2020; (20):65-8.

44. Moser DA, Glaus J, Frangou S, et al. Years of life lost due to the psychosocial consequences of COVID19 mitigation strategies based on Swiss data. European Psychiatry. 2020; 63(1):1-7.

45. Chandan JS, Taylor J, Bradbury-Jones C, et al. COVID-19: a public health approach to manage domestic violence is needed. Lancet Public Health. 2020; 5(6):e309.

46. Anurudran A, Yared L, Comrie C, et al. Domestic violence amid COVID-19. Int J Gynaecol Obstet. 2020; 255-6.

47. Neil J. Domestic violence and COVID-19: Our hidden epidemic. Aust J Gen Pract. 2020; 49(supl25):1.

48. Vieira PR, Garcia LP, Maciel ELN. The increase in domestic violence during the social isolation: what does it reveals? Rev. Bras. Epid. 2020; 23:e200033.

49. Telles LEdB, Valenca AM, Barros AJS, et al. Domestic violence in the COVID-19 pandemic: a forensic psychiatric perspective. Braz J Psychiatry. 2020; (S1516):44462020005015211.

50. Organização das Nações Unidas. Mulheres Brasil. Gênero e COVID-19 na América Latina e no Caribe: dimensões de gênero na resposta. Brasília: ONU Mulheres Brasil. [internet]. [acesso em 2020 jul 24]. Disponível em: http://www.onumulheres.org.br/wp-content/uploads/2020/03/ONU-MULHERES-COVID19_LAC.pdf.

51. Bradbury-Jones C, Isham L. The pandemic paradox: The consequences of COVID-19 on domestic violence. J Clin Nurs. 2020; 29(13-14):2047-9.

52. Boserup B, McKenney M, Elkbuli A. Alarming trends in US domestic violence during the COVID-19 pandemic. Am J Emerg Med. 2020; 38(12):2753-2755. 
53. Marques ES, Moraes CLd, Hasselmann MH, et al. Violence against women, children, and adolescents during the COVID-19 pandemic: overview, contributing factors, and mitigating measures. Cad. Saúde Pública. 2020; 36(4):e00074420.

54. Bellizzi S, Nivoli A, Lorettu L, et al. Violence against women in Italy during the COVID-19 pandemic. Int J Gynaecol Obstet. 2020; 150(2):258-9.

55. Ghoshal R. Twin public health emergencies: Covid-19 and domestic violence. Indian J Med Ethics. 2020; $\mathrm{V}(3): 1-5$

56. Mohler G, Bertozzi AL, Carter J, et al. Impact of social distancing during COVID-19 pandemic on crime in Los Angeles and Indianapolis. J Crim Justice. 2020; 68:101692.

57. Rossi FS, Shankar M, Buckholdt K, et al. Trying Times and Trying Out Solutions: Intimate Partner Violence Screening and Support for Women Veterans During COVID-19. J Gen Intern Med. 2020; 35; (9):2728-31.

58. Bohoslavsky JP, Rulli M. Covid-19, International Financial Institutions and the Continuity of Androcentric Policies in Latin America. Rev. Estud. Femin. 2020; 28(2):e73510.

59. Ruiz-Pérez I, Pastor-Moreno G. Medidas de contención de la violencia de género durante la pandemia de COVID-19. Gaceta Sanitaria. 2020.

60. Sacco MA, Caputo F, Ricci P, et al. The impact of the Covid-19 pandemic on domestic violence: The dark side of home isolation during quarantine. Med Leg J. 2020; 88(2):71-3.

61. Bouillon-Minois J-B, Clinchamps M, Dutheil F. Coronavirus and Quarantine: Catalysts of Domestic Violence. Viol. Against Women. 2020; (0):1-3.

62. Mazza M, Marano G, Lai C, et al. Danger in danger: Interpersonal violence during COVID-19 quarantine. Psychiatry Res. 2020; 289:(113046).
63. Mengin A, Alle MC, Rolling J, et al. [Psychopathological consequences of confinement]. Encephale. 2020; 46(3S):S43-S52.

64. Blackman A, Ibáñez A, Izquierdo A, et al. A política pública de combate à Covid-19: Recomendações para a América Latina e o Caribe [internet]. [acesso em 2020 jul 24]. Disponível em: https://publications. iadb.org/publications/portuguese/document/A-politica-publica-de-combate-a-Covid-19-Recomendaces-para-a-America-Latina-e-o-Caribe.pdf.

65. Instituto Brasileiro de Geografia e Estatística. Coordenação de População e Indicadores Sociais. Síntese de Indicadores Sociais: uma análise das condições de vida da população brasileira: 2019. Rio de Janeiro: IBGE; 2019.

66. Zamarro G, Perez-Arce F, Prados MJ. Gender Differences in the Impact of COVID-19: Working Paper.; 2020 [internet]. [acesso em 2020 jul 24]. Disponível em: https://ktla.com/wp-content/uploads/ sites/4/2020/06/ZamarroGenderDiffImpactCOVID-19_061820-2.pdf.

67. Boniol M, McIsaac M, Xu L, et al. Gender equity in the health workforce: analysis of 104 countries: World Health Organization [internet]. [acesso em 2020 jul 24]. Disponível em: https://www.who.int/hrh/resources/gender_equity-health_workforce_analysis/en/.

68. Machado MH, Oliveira EdSd, Moyses NMN. Tendências do mercado de trabalho em saúde no Brasil. O trabalho em saúde: abordagens quantitativas e qualitativas. 2011; (1):103-16.

69. Adams JG, Walls RM. Supporting the health care workforce during the COVID-19 global epidemic. Jama. 2020; 323(15):1439-40.

70. Huang L, Lin G, Tang L, et al. Special attention to nurses' protection during the COVID-19 epidemic. 2020; 24(1):120.

71. Ran L, Chen X, Wang Y, et al. Risk factors of healthcare workers with corona virus disease 2019: a retrospective cohort study in a designated hospital 
of Wuhan in China. Clinic. Infect. Diseases. 2020; 71(16):2218-2221.

72. Rolim-Neto ML, Almeida HG, Esmeraldo JDa, et al. When health professionals look death in the eye: the mental health of professionals who deal daily with the 2019 coronavirus outbreak. Psychiatry Research. 2020; (288):112972.

73. Bai Y, Wang X, Huang Q, et al. SARS-CoV-2 Infection in Health Care Workers: A Retrospective Analysis and Model Simulations of a Nosocomial Outbreak. SSRN Journal [preprint] [internet]. 2020 [acesso em 2020 jul 24]. Disponível em: https://papers.ssrn. com/sol3/papers.cfm?abstract_id=3566208.

74. Hankivsky O, Kapilashramim A. A.Beyond sex and gender analysis: an intersectional view of the COVID-19 pandemic outbreak and response. In: Gender and Women's Health Unit CfHE, Melbourne School of Population and Health Equity. Victoria: University of Melbourne; 2020.

75. Machado MH, Oliveira EdSd, Lemos WR, et al. Mercado de trabalho em enfermagem no âmbito do SUS: uma abordagem a partir da pesquisa Perfil da Enfermagem no Brasil. Divulg Saude Debate. 2016; (56):52-69.

76. Spoorthy MS. Mental health problems faced by healthcare workers due to the COVID-19 pandemic-a review. Asian J. Psyc. 2020; (51):102119.

77. Pappa S, Ntella V, Giannakas T, et al. Prevalence of depression, anxiety, and insomnia among healthcare workers during the COVID-19 pandemic: A systematic review and meta-analysis. Brain Behav Immun. 2020; (88):901-7.

78. El-Hage W, Hingray C, Lemogne C, et al. Les professionnels de santé face à la pandémie de la maladie à coronavirus (COVID-19): quels risques pour leur santé mentale? L’Encephale. 2020; 46(35):S73-S80.

79. Vindegaard N, Benros ME. COVID-19 pandemic and mental health consequences: systematic review of the current evidence. Brain Behav Immun. 2020; (89):531-542.
80. Özdin S, Bayrak Özdin Ş. Levels and predictors of anxiety, depression and health anxiety during COVID-19 pandemic in Turkish society: The importance of gender. Inter. J. Social Psychiatry. 2020; 66(5):504-11.

81. Wang Y, Di Y, Ye J, et al. Study on the public psychological states and its related factors during the outbreak of coronavirus disease 2019 (COVID-19) in some regions of China. Psychol. Health Med. 2020; 26(1):1-10.

82. St-Denis X. Sociodemographic Determinants of Occupational Risks of Exposure to COVID-19 in Canada. Canadian Rev. of Sociol. [internet]. 2020 [acesso em 2020 jul 24]. Disponível em: https://osf.io/preprints/socarxiv/nrjd3/.

83. Kramer A, Kramer KZ. The potential impact of the Covid-19 pandemic on occupational status, work from home, and occupational mobility. J. of Vocat. Behav.2020; (119):4.

84. Goes EF, Ramos DdO, Ferreira AJF. Desigualdades raciais em saúde e a pandemia da Covid-19. Trab. Edu. Saúde. 2020; 18(3).

85. Hawkins D. Differential occupational risk for COVID-19 and other infection exposure according to race and ethnicity. Am. J. Ind. Med. 2020; 63:817-20.

86. Shimazu A, Nakata A, Nagata T, et al. Psychosocial impact of COVID-19 for general workers. J. Occup. Health. 2020; 62(1):1.

87. Power K. The COVID-19 pandemic has increased the care burden of women and families. Sustainab. Scien., Pract. Polic. 2020; 16(1):67-73.

88. Stokes JE, Patterson SE. Intergenerational Relationships, Family Caregiving Policy, and COVID-19 in the United States. J. Aging. Soc. Polic. 2020; 32(45):416-24.

89. Flaxman S, Mishra S, Gandy A, et al. Estimating the number of infections and the impact of non-pharmaceutical interventions on COVID-19 in European countries: technical description update [preprint] 
[internet]. [acesso em 2020 jul 20]. Disponível em: https://www.imperial.ac.uk/mrc-global-infectious-disease-analysis/covid-19/report-13-europe-npi-impact/.

90. Ferguson N, Laydon D, Nedjati-Gilani G, et al. Report 9: Impact of non-pharmaceutical interventions (NPIs) to reduce COVID19 mortality and healthcare demand. Imp. Colleg. London. 2020; (10):77482.

91. Cohen J, Kupferschmidt K. Countries test tactics in 'war'against COVID-19. Science. 2020; 367(6484):2.

92. Mahase E. Covid-19: schools set to close across UK except for children of health and social care workers. BMJ. 2020; (368):1.
93. Kent EE, Ornstein KA, Dionne-Odom JN. The family caregiving crisis meets an actual pandemic. J. Pain Sympt. Manag. 2020; 60(1):e66-e9.

Recebido em 29/07/2020

Aprovado em 26/09/2020

Conflito de interesses: inexistente

Suporte financeiro: Góes EF é financiada pelo National Institute for Health Research (NIHR) Global Health Research Programme (GHRG/16/137/99). Aquino EML tem Bolsa de Produtividade em Pesquisa do Conselho Nacional de Desenvolvimento Científico e Tecnológico (CNPq) (Processo no 306295/2017-2) 